\title{
L'échec dans Qué sola estás Maité d'Arquímedes González : mensonge et désillusion au Nicaragua
}

Nathalie Besse

\section{(2) OpenEdition}

1 Journals

Édition électronique

URL : https://journals.openedition.org/cher/12049

DOI : $10.4000 /$ cher. 12049

ISSN : 2803-5992

Éditeur

Presses universitaires de Strasbourg

Édition imprimée

Date de publication : 30 juin 2012

Pagination : 39-49

ISBN : 978-2-35410-046-9

ISSN : 1968-035X

\section{Référence électronique}

Nathalie Besse, «L'échec dans Qué sola estás Maité d'Arquímedes González : mensonge et désillusion au Nicaragua », reCHERches [En ligne], 8 | 2012, mis en ligne le 21 février 2022, consulté le 24 février 2022. URL : http://journals.openedition.org/cher/12049; DOI : https://doi.org/10.4000/cher.12049

\section{(c) (i) (8)}

Ce(tte) œuvre est mise à disposition selon les termes de la Licence Creative Commons Attribution Pas d'Utilisation Commerciale - Partage dans les Mêmes Conditions 4.0 International. 


\title{
L'échec dans Qué sola estás Maité d'Arquímedes González: mensonge et désillusion au Nicaragua
}

\author{
NATHALIE BESSE \\ Université de Strasbourg
}

Il se peut que nous soyons, nous autres écrivains, des survivants ou, au contraire, des guetteurs de l'aube. Richard Millet, Désenchantement de la littérature

\begin{abstract}
Qué sola estás Maité (2007) est le deuxième roman d'Arquímedes González, jeune auteur nicaraguayen primé pour ses contes. La solitude dont il est question dans le titre révèle l'échec d'une vie, celui de la protagoniste, dont les relations «manquées» avec les siens s'inscrivent dans un plus vaste échec, national cette fois, celui du Nicaragua.

Ainsi verrons-nous comment le mensonge et la désillusion qui amorcent l'échec avec les proches, le doivent à la duplicité de Maité, un personnage allégorique à bien des égards, assimilé métaphoriquement à des cataclysmes de l'Histoire du petit pays tels que la révolution sandiniste ou l'ouragan Mitch. Ces épisodes tragiques sont d'ailleurs matière à dénoncer la corruption légendaire, sinon intrinsèque, d'un Nicaragua né de l'usurpation.
\end{abstract}

\section{Mensonge et désillusion: l'échec de la relation}

Qué sola estás Maité se donne d'abord à lire comme l'histoire d'une rupture entre la protagoniste et Miguel après l'adultère de celle-ci. Cet échec 
de la relation qui caractérise le couple en soi, altère tout autant leurs familles respectives elles aussi menacées par le mensonge et la désunion. À cet égard, les familles divisées de Maité et de Miguel présentent nombre d'analogies: «immoralité» du parent de sexe opposé et déception de l'enfant qui influera sur sa relation de couple future.

En effet, Maitéressent une profonde aversion pour son père qu'elle surprend au petit matin alors qu'il se masturbe devant un film pornographique: «[...] pero lo peor vino cuando lo encontraste aquella madrugada haciendo esa cochinada, ese ser que para ella era la persona más centrada, moral y sincera en este mundo, se desvaneció Maité, cuando descubriste lo que hacía frente al televisor» (124); Miguel est traumatisé quant à lui après avoir surpris sa mère en flagrant délit d'adultère: "te marcó la vida, como el sello que le ponen al ganado. [...] sería tu ruina con las mujeres» (82).

Tous deux profondément marqués par la fausseté du parent dont ils avaient une image valorisée, rechercheront dès lors en l'autre le père idéal ou la mère irréprochable, et ne se tromperont que davantage dans la relation qu'ils imprégneront de leur amertume et leur espoir. Maité, écœurée par l'hypocrisie morale du père, «un hombre tan recto que recordaba los valores, la familia y los sacramentos» (124), n'aura de cesse de s'opposer à lui et de le chercher dans les autres hommes:

buscabas la figura paterna que no tuviste porque te dejó sin respuestas e hiciste preguntas a Miguel y a Fernando para que llenaran esos desequilibrios que nacieron cuando encontraste a tu padre frente al televisor [...], trastocando tu vida. Fue él quien te sembró la semilla de la demencia que hoy padecés... (128).

Si Maité attend inconsciemment de Miguel et Fernando (qui a vingt ans de plus qu'elle) qu'ils compensent les manques provenant du père, Miguel devient pour sa part ce misogyne qui soupçonne en toute femme une traîtresse, et qui cherche une "mère» en Maité qu'il espère différente des autres (107). Le rapport à tel ou tel parent conditionne le rapport au sexe opposé: en l'occurrence, la «cochinada» d'un des parents révélée brutalement - le même terme est employé dans les deux histoires (83 et 124) - détermine certains comportements dans le couple qui mènent à l'échec: Miguel provoque la sensation d'étouffement de Maité par sa possessivité, mais celle-ci suscite la jalousie de Miguel par son indépendance et un sens de la rébellion qui émanent du vécu de l'enfance.

Il semble que soit à l'œuvre ici une névrose d'échec: à vouloir conjurer l'épisode douloureux de l'enfance, les personnages, qui en sont d'une 
certaine façon prisonniers, se mettent malgré eux en situation de reproduire la frustration première, ce qui les conforte dans la vérité de cette blessure et les en disculpe en leur faisant projeter la culpabilité de l'échec sur l'autre.

Cette incompréhension, cet échec de la communication parce que chacun attend de l'autre qu'il réécrive une histoire qui n'est pas la sienne, qu'il soit autre qu'il n'est, et le met ainsi en porte-à-faux, apparaît de façon frappante dans les sept chapitres extrêmement courts de dialogues aux répliques très brèves et aux silences éloquents - ces séquences contrastent d'ailleurs avec les quinze chapitres «logorrhée» décrivant l'examen de conscience de Maité ou de Miguel, et s'adonnant à un pamphlet en règle de l'Histoire politique au Nicaragua.

Si tout amour est «engaño» d'après Maité elle-même (55), Miguel en vient à penser que le leur «está como el lago de Managua, envenenado, podrido, hediondo y sin salvación» (87): amour cloaque donc, sale et corrompu comme les eaux croupies de la capitale. Cette lucidité redoutable coexiste pourtant avec un besoin de rester dans l'illusion par peur d'une solitude qui rapproche de la mort intérieure; en effet, Miguel songe, après l'abandon: «Me han dejado muerto en vida» (150).

Échec larvé de la relation dans le mensonge que Maité raconte à Miguel et que ce dernier se raconte à lui-même, échec manifeste quand la vérité éclate: le même scénario définit la relation de Maité à ses parents auxquels elle cache, de crainte du rejet, une grossesse fruit de l'adultère. Lorsque la mère l'apprend, la déception est exprimée de la façon la plus patente qui soit: «nos mentiste», «nos decepcionaste» (38), d'où la rupture définitive avec la famille qui la bannit et que Maité abandonnera à son tour quand ses parents seront touchés par la maladie et la mort, comme le montre cette phrase d’une cruelle symétrie: «¡No volvás!...Y Teresita jamás volvió» (38).

Il apparaît clairement dans ces exemples que l'échec trouve sa source dans le mensonge, puis dans la désillusion qui en découle pour la personne trahie. Cette duplicité, cause de l'échec, s'avère un trait définitoire de Maité, une conséquence malheureuse de la dualité que contient et annonce son prénom.

\section{Duplicité et cataclysme: Maité, un personnage allégorique du Nicaragua}

Maité est le diminutif ou la condensation de María Teresa, une identité moins vécue sur le mode de la synthèse que sur celui du dualisme, voire d'une forme de déchirement à en juger par certains débats intérieurs: «está 
dividida. Es capaz de ser dos personas» (148). C'est l'ambivalence, sinon la scission, qui définit Maité, cette dualité rimant avec duplicité et c'est précisément parce que Maité est un personnage du mensonge qu'elle se façonne, sans en avoir conscience, en personnage de l'échec.

Lorsqu' elle choisit de mettrefin à sa relation avec Miguel, ce bouleversement est métaphorisé par un cataclysme: en effet, Maité est mise en parallèle puis identifiée, dans les quelques lignes liminaires des dialogues, avec l'ouragan Mitch qui s'abattit sur le Nicaragua en 1998. Maité devient ce "peligroso huracán» (39) qui anéantit tout sur son passage, et il n'est pas anodin que la mort du couple qu'elle provoque en quittant le foyer conjugal coïncide avec la mort de Miguel en mission à Posoltega où l'ouragan fait des ravages: «Es el día que Maité se desata como el peor huracán» (147).

Mais les analogies ne s'arrêtent pas là. Maité se voit assimilée à plusieurs reprises au tourbillon létal de 1998 comme la révolution sandiniste est comparée au tremblement de terre historique du 23 décembre 1972 qui dévasta Managua. Cette métaphore commune du séisme rapproche une Maité «révolutionnant» son existence des révolutionnaires qui renversent un ancien régime. Sa relation asphyxiante avec Miguel apparaît comme sa dictature si l'on ose dire, et son départ avec Fernando comme son «monde nouveau ».

Cela dit, l'émancipation de Maité et la libération sandiniste s'avèrent des victoires à la Pyrrhus incitant à se demander si cela valait la peine, une question récurrente dans les fictions nicaraguayennes de la période dite post-utopique. Mais là n'est pas l'unique critique à peine voilée d'un roman par ailleurs percutant dans ses énoncés: en effet, cette Maité qui trompe Miguel évoque l'image bien connue d'une révolution trahissant ceux qui l'aimaient, à savoir le peuple. Dans ce couple sali par le mensonge comme l'aura été l'aventure sandiniste, Maité incarne tout autant le peuple tenté par la révolution que le mensonge qu'elle a représenté, tandis que Miguel symbolise le peuple trompé.

Aussi bien, le terme de "corrupción» s'agissant de l'adultère de Maité, associe-t-il sa tromperie à la corruption du pays évoquée dans le même paragraphe (91). Le mensonge de Maité contient l'allégorie d'une plus vaste trahison:

Pueblo corrupto, pueblo de ladrones, mentirosos... ¿Y vos? ¿No sos parte de este pueblo? ¿No has cometido los mismos errores que criticás? [...] Sos lo que le ha pasado a este país en los últimos treinta años, liberado de una dictadura, gozó de la libertad y oportunidad de rehacerse con una revolución que por desgracia rompió esquemas y al final, terminó por traicionarse (60). 
Mais plus qu'un parallèle, c'est une corrélation qui se dégage des lignes de Qué sola estás Maité si l'on en croit l'idée de la protagoniste selon laquelle la société influe sur les individus: "porque aquí nos enseñan a tener doble cara $[\ldots]$, nos enseñan que robar es la necesidad básica insatisfecha, la infidelidad, lo normal, la mentira, la práctica cotidiana, y el odio y el rencor, lo que nos alimenta» (125). Maité paraît se dédouaner de sa traîtrise en arguant d'un contexte socio-politique défavorable. Miguel inculpe lui aussi ce Nicaragua décentré qui devient une circonstance atténuante en cas d'échec personnel: "Ni María ni yo nos salvamos de esta desgracia de vivir en un lugar ambiguo y perturbado» (142). Le père de Miguel accuse quant à lui sans la moindre ambiguïté: «Fue esa puta revolución la que nos trastornó, la que nos volvió locos...» (138).

La quête du bonheur qui motive la révolution sociale et la révolution individuelle, mène en l'occurrence à l'échec auquel elle espérait remédier. Quant à la plénitude, ou l'intensité, que connaissent Maité dans les bras de son amant ou les «muchachos» entrant triomphalement à Managua au beau milieu de la liesse populaire, elle ne saurait durer qu'un temps infime avant de laisser à nouveau place au réel. Et sans doute le réveil est-il d'autant plus douloureux que la transe aura été extatique. Au lendemain de ces tentations et tentatives de vivre plus pleinement, Maité, ainsi que les révolutionnaires au pouvoir, paient le prix du mensonge, tandis que le peuple a la gueule de bois. L'échec d'une vie s'inscrit ici dans l'échec d'une Histoire nationale.

Si Cioran estime que «l'échec, toujours essentiel, nous dévoile à nousmêmes» (1281), c'est pourtant une Maité face à son propre vide que dépeint le roman, une Maité seule après avoir trompé les siens, "derrotada», "vencida» (119), qui sombre dans la dépression. "Comment une vie toute faite d'échecs n'éprouverait-elle pas le sentiment de l'Échec irrémédiable?» demande Jean Lacroix (1965: 113) qui rappelle dès le seuil de son ouvrage que «les échecs portent sur les intentions que nous avons et l'Échec sur l'Intention que nous sommes" (1). Maité s'éprouve nécessairement comme une intention, un projet de réalisation, une exigence d'être, ce qui pose le problème du libre arbitre - si tant est que l'on soit libre de ses démons et que les choix conscients et raisonnables ne soient pas en réalité l'expression d'une névrose d'échec, ou que le principe de réalité auquel on croit s'en remettre ne masque pas un irréductible principe de plaisir comme le montre le chemin malheureux que se trace Maité en proie à un désir dont la nature même est de ne jamais pouvoir être satisfait. 
Pour exister, se réaliser, elle doit courir le risque de l'échec parce qu'elle n'est pas toute faite mais à faire, sans jamais devenir cependant tout ce qu'elle pourrait être, ce qui amène Nicolas Grimaldi à affirmer: "Au bout de toute vie, chacun ne s'éprouve ainsi que comme le survivant de tant d'autres moi qu'il a laissé périr. Dans l'ordre de la représentation, cet insurmontable inachèvement et cette incomplétude font de toute existence une sorte d'échec métaphysique» (2003: 205).

Si le philosophe fait donc de l'échec un aspect inhérent à toute existence, il nous faut néanmoins rappeler que dans l'histoire de Maité, ce sont plus ses décisions erronées (égoïstes ou intempestives), son insincérité, mais également les morts qui jalonnent son destin accidenté, qui y sont pour quelque chose dans ce sentiment d'un vaste échec existentiel: à la rupture avec son époux et ses propres parents, s'ajoutent la mort de Miguel, la perte de l'enfant de Fernando lors d'une fausse couche, puis sa stérilité, le décès de Fernando enfin.

Nous avons assez dit à quel point les personnages de ce roman sont explicitement allégoriques et à mettre en parallèle, ou en symbiose, avec les événements du petit pays: aussi comprenons-nous que, outre un Miguel trompé par Maité qui rappelle le peuple abusé par la révolution, la mort de l'enfant fruit de la libération peut symboliser l'absence de futur d'une révolution condamnée - la stérilité de Maité ou la mort de Fernando vont dans le même sens - ou métaphoriser les nombreux morts de la guerre civile.

On peut s'interroger également sur cette inquiétante étrangeté que ressent Maité lorsque, avant de quitter le pays avec Fernando, elle parcourt en taxi la Managua de son enfance et de sa jeunesse en songeant: "como si esos lugares no la reconocieran" (162). À l'instar de ce Nicaragua qui très tôt n'a pas reconnu la révolution pour laquelle il avait combattu? Précisément parce que celle-ci s'est bien vite détournée du peuple, comme Maité abandonne les siens avec l'illusion d'avancer vers un monde meilleur. Car outre les mensonges que Maité sert à ses proches, sévit le mensonge envers soi-même que sanctionnera fatalement un réel reprenant ses droits.

L'excipit de Qué sola estás Maité, qui relate cette fuite en avant au Costa Rica avec son amant, pose le problème de cette "auto-mystification» dans un paragraphe dense où abondent les négations pour dire autant de dénégations, parmi lesquelles un consternant «aquí no pasó nada» (163) qui n'est pas sans rappeler la façon dont les politiciens corrompus et le peuple blasé feignent respectivement l'innocence et l'indifférence, ce sur 
quoi le roman ne manque pas d'ironiser à plusieurs reprises. De la déloyauté au déni, le Nicaragua s'embourbe dans un mensonge présenté comme consubstantiel à son Histoire.

\section{Nicaragua et corruption: l'échec de toute une Histoire}

Les paragraphes corrosifs portant sur le Nicaragua, ses dirigeants, son Histoire, semblent relater une chronique du vol ou de la malversation, qui fait de la corruption une sorte d'atavisme ou de fatalité prenant valeur de loi: "Han legalizado la corrupción» (91). Celle-ci explique que dans ce récit, comme dans d'autres romans nicaraguayens contemporains, soient abondamment exploités le thème excrémentiel et la métaphore sordide du porc. Pouvoir, petit pays, tout est souillure: «Es mejor irse a la mierda y volver a Nicaragua» (54), "Este desgraciado país de mierda» (162), ou encore «El país convertido en porqueriza» (27), ce dernier terme renvoyant à celui de "cochinada" qui définissait aussi bien l'adultère de la mère de Miguel que l'onanisme du père de Maité (83 et 124). Dans ce pays de porcs, qui symbolisent des politiciens salement engraissés, les familles de "porcs» - également salies par leurs turpitudes - de Miguel et de Maité amènent ces deux derniers à vomir après le flagrant délit (82 et 124): faut-il en déduire que la nausée est ce qu'inspirent à l'auteur la corruption proverbiale du Nicaragua et ses trahisons politiques?

Cela étant, si Qué sola estás Maité fait de la corruption et de la trahison un leitmotiv historique, ce sont le régime sandiniste et le gouvernement d'Arnoldo Alemán qui reçoivent les critiques les plus acerbes dans ce roman du désenchantement qui incrimine tout autant les utopies de gauche qu'une prétendue démocratie masquant mal un néo-libéralisme prédateur et déprédateur.

Nous nous souvenons que la révolution, comparée à un volcan, est rapprochée du cataclysme de 1972 qui laissa derrière lui 40000 morts (30 et 76), presque autant que les 50000 morts de la révolution dont le résultat fut, si l'on en croit Maité, «joder al país y sumirlo en el cataclismo que ni guerras ni terremotos lograron» (33) comme si elle avait représenté le pire chaos de l'Histoire du pays. L'échec est directement corrélé à l'idéologie qu'elle véhiculait: "una gran nación comunista. Y con esto empezó el fracaso» (32). Cet échec des utopies sociales n'absout pas chacun de ses dirigeants dont Qué sola estás Maité s'ingénie à ridiculiser l'autorité, notamment au 
moyen de la ritournelle des «nueve Comandantes enanos» démythifiés sans ambages: "pasaron de ser míticos Comandantes a bíblicos ladrones» (24).

Les attaques se font plus virulentes lorsque ces «rufianes y saqueadores" (24) dont le roman dénonce les mensonges, sont comparés par Maité aux pires bourreaux de l'Histoire: «[...] maravillas del comunismo, cuando lo que aquí hay es un campo de concentración como en Alemania, ahí eran los nazis, éstos aquí son los revolucionarios que nos tienen secuestrados» (24). Après l'image du cataclysme dont émanait déjà la notion de chaos, les ténèbres de l'holocauste renvoient à nouveau à la mort, ajoutant à la tragédie l'idée du Mal, de la folie des hommes qui, entremêlée à leurs passions, écrit violemment l'Histoire.

Cette vision cinglante d'une révolution déshumanisée, bâtissant un monde en deçà de l'humain, dit toute l'amertume de la désillusion: "¿Hubo alguna vez revolución en este país? [...], parece que no, que lo que hubo fue una desilusión colectiva, un engaño o un atraco simultáneo a las esperanzas. La revolución produjo muerte, pobreza, odio, rencor, desgracia y tristeza » (59). On ne saurait affirmer plus cuisant constat d'échec.

Pour autant, la défaite de la révolution ne signe pas l'avènement de temps meilleurs puisque la farce éhontée que représentera la présidence d'Arnoldo Alemán ne fera qu'accentuer la rupture de confiance et d'espoir dans la politique. Satiriquement surnommé «El Excelentísimo», ce dirigeant «engraissé» dont on souligne à dessein l'obésité croissante, deviendra vite un nouvel archétype de la corruption: «El aumento de su capital fue igual que su peso. [...] De robusto pasó a ser barril lleno además de grasa, de dinero » (109).

Personnage gargantuesque qui paraît ingurgiter le Nicaragua, il rappelle à tous l'ancien dictateur qui régnait sans partage sur le petit pays: «Ser dueño de Nicaragua a como lo fue la familia Somoza» (109); et comme lui lors du tremblement de terre de 1972, il détourne l'aide internationale attribuée après les ravages de l'ouragan Mitch. Lorsque la Justice s'en mêle, il déploie toute une "comédie» du pouvoir, ses mensonges révélant un cynisme innommable face à la tragédie dans laquelle plusieurs milliers de personnes ont péri; sans omettre l'indécence morale de sa richesse ostentatoire, ces Chevrolet Suburban rutilantes dans lesquelles pavane le cortège de sa «cour» ministérielle elle aussi grassement rémunérée pour ses quelques services et ses nombreuses complicités. Le roman brocarde cet autre ouragan qui emporte les dons: «la glotonería de los funcionarios» (158), comme si la corruption dévastait autant qu'un cataclysme. 
Mais au Nicaragua, le pouvoir peut s'approprier frauduleusement des sommes colossales sans être inquiété d'aucune façon: sur les trente-six fonctionnaires dénoncés pour avoir volé 450 millions de dollars, seul Arnoldo Alemán eut quelques déboires avec la Justice qui se contenta de le mettre sous surveillance, car où règne la corruption, triomphe l'injustice, tandis qu'échouent la société des hommes et le pouvoir qui est censé la préserver. Dans la même veine, lorsqu'Arnoldo Alemán pactise avec l'exprésident sandiniste Daniel Ortega comparé à un hors-la-loi - «ha pactado con aquellos forajidos revolucionarios» (142) -, et qu'ils détournent l'argent qui pourrait servir à l'éducation et la santé, ce qui est une façon implicite d'associer pouvoir et mort, même le Comité d'Éthique du gouvernement couvre le méfait (142).

Les Nicaraguayens eux-mêmes se désintéressent bien vite de ce brigandage des hautes sphères, accoutumés qu'ils sont à une corruption «institutionnalisée»: «Es la robocracia, la impunidad civilizada» (141); une impunité héritée de l'Histoire du petit pays qui naît de l'usurpation dès la Conquête: "Hemos nacido para robar y que nos roben. [...] Somos un pueblo que nació del robo" (140). Vol et corruption s'avèrent inhérents à l'Histoire viciée d'un peuple «vache à lait» dans un pays dont les pensées de Miguel vantent ironiquement un singulier "way of life», un surprenant «rêve américain »:

El Excelentísimo Presidente de la Respública, perdón, República, es muestra de cómo se trepa desde vende huevos a hacendado y millonario [...], ni Bill Gates lo supera, Nicaragua es el sueño latinoamericano, no se vayan a Estados Unidos a limpiar inodoros bola de pendejos, aquí se pueden cumplir sus ilusiones, basta con pagar coima al Excelentísimo y a los Comandantes enanos y se resuelve (142).

Le village de Posoltega dont les maisons sont comparées à de fragiles boîtes d'allumettes, où il n'y a pas d'hôpital et pas véritablement de routes, ce qui amène à se demander qu'est devenu tout l'argent destiné à sa construction, est la preuve tangible du vol et de la tromperie. Aussi bien, juste avant que Mitch ne dévaste, le 29 octobre 1998, ce lieu hautement symbolique - sur les flancs du volcan Casita dont la coulée de boue devait tout ensevelir -, l'ouragan qui approche semble-t-il «la mala conciencia del mundo» (153).

$\mathrm{Du}$ reste, est-ce le monde entier qui peut avoir mauvaise conscience si l'on considère que Mitch est mis en rapport direct avec la folie des hommes qui ont perturbé le rythme de la nature: « el país de rodillas por el ciclón más 
poderoso de la historia reciente, efectos de El Niño, por la contaminación, por haber destruido la naturaleza " (158). L'exploitation abusive des ressources naturelles s'applique également aux individus dans un monde gangrénépar tout un système, vraisemblablement le néo-capitalisme: "Así sucede en el mundo en donde lo que más abunda es la globalización de la esclavitud y la explotación» (46). Ainsi, l'Amérique latine subit-elle un système inhumain danslequel le capital prévaut sur la personne: «desechan a los empleados como si fueran latas o cajas de cartón» (45). Le roman n'épargne pas même le Costa Rica qui voit son image de bon élève latino-américain se ternir dans les pensées de Maité - celle-ci semblant lui reprocher en outre la même hypocrisie bien-pensante que son père - : «la Suiza de Centroamérica que al final, descubriste que era la más puerca porque durante los últimos años sus tres ex presidentes vendieron el país a las transnacionales» (48).

Les abus dénoncés au Nicaragua sont commis en d'autres lieux, et ce qui est mis en accusation ici, c'est en définitive l'homme qui, de par sa nature même, s'avère un loup pour l'homme entre autres nombreuses laideurs comme l'affirment des lignes qui, après avoir rappelé la prédisposition humaine à la corruption et à la transgression, dépeignent:

este mundo lleno de miserias, en este rincón del universo donde los seres no caminan, reptan por ser tan bajos e inescrupulosos, tan vanidosos y arrogantes, egoístas y mezquinos, mentirosos y embusteros, lameculos e hipócritas por querer a toda costa lo que el otro tiene, por robar y destruir lo poco de sinceridad que queda en algunos refugios [...] (91).

Comment un monde constitué de telles créatures, humaines trop humaines, ne serait-il pas voué à l'échec? Le Nicaragua souffrirait pourtant davantage d'après Maité qui semble en faire la victime d'un destin implacable, sinon sadique: «desgracia tras desgracia, Nicaragua, el lugar perfecto para los experimentos más crueles del tormento » (161). Et une fois encore l'idée implicite d'une malédiction. Certes, ce sont les hommes qui ont fait l'Histoire et qui sont donc responsables pour une bonne part des maux du petit pays, mais la prégnance de cette Histoire ou du contexte socio-politique qui en découle, n'agit-elle pas à son tour et en retour sur les hommes entraînés malgré eux dans le tourbillon qu'ils ont provoqué, "prisonniers» - parfois consentants, il est vrai - d'un puissant cercle vicieux? Entre responsabilité de chacun et fatalité, il semble que Qué sola estás Maité, qui donne à voir l'engrenage, dresse un constat d'échec «total».

Si la cause de l'échec réside dans le mensonge, l'une de ses conséquences est la solitude, celle de Maité annoncée dans l'intitulé même du roman, mais 
également celle d'un Nicaragua pour ainsi dire abandonnépar ses "pères", non pas protecteurs mais prédateurs, non pas donneurs des lois mais transgresseurs, des pères qui, tout au long de l'Histoire, n'ont jamais incarné une autorité respectable mais ont jeté le discrédit sur le pouvoir qui, loin de se faire garant de l'ordre, aura fomenté une forme insidieuse d'anarchie dans le pays. C'est un Nicaragua en ce sens déstructuré et orphelin qui émerge d'entre les lignes, vraisemblablement en quête du père, ou de «re-pères", à l'instar de Maité. Histoire de l'échec, échec de l'Histoire, Qué sola estás Maité dénonce les racines du mal d'un petit pays «avorté», empêché de s'exaucer, dont l'échec politique et social étouffe l'existence.

L'une des questions qui traverse en filigrane ce récit pourrait bien être "comment être soi?». Car le meilleur antidote contre l'échecn'est-il pas précisément d'être, ou de devenir, ce que l'on est ? Maité démontre par l'absurde que se réaliser c'est transcender la dualité, parvenir à une intégrité qui est aussi sincérité. Loin de toute duplicité, de ce mensonge présenté comme un véritable fléau, n'est-ce pas une éthique que prône, par déduction, Qué sola estás, Maité? Cette fiction ne différerait pas en cela d'autres romans nicaraguayens modernes qui, pour la plupart, dénoncent avec force la corruption ambiante et plaident, plus ou moins explicitement, pour une nouvelle éthique. Ainsi le roman qui nous occupe recèlerait-il, au-delà de la condamnation et d'un constat d'échec relativement fataliste, l'espoir de troubler les esprits assoupis. De susciter un éveil.

\section{Bibliographie}

Cioran, 1995, «De l'inconvénient d'être né», in Euvres, Paris, Quarto Gallimard. Gianni, Silvia, 2007, «El porvenir del pasado: la novela nicaragüense entre historia canonizada, lucha representacional y pluralidad de lecturas», Istmo $\mathrm{n}^{\circ} 15$.

González, Arquímedes, 2007, Qué sola estás Maité, Managua, Anamá Ediciones.

González, Arquímedes, site web del'auteur : http://arquimedesgonzalez.blogspot.com/ Lacroix, Jean, 1965, L'échec, Paris, PUF.

Lacroix, Jean (ss dir.), 1968, Les hommes devant l'échec, Paris, PUF.

Grimaldi, Nicolas, 1998, Bref traité du désenchantement, Paris, PUF.

Grimaldi, Nicolas, 2003, Traité des solitudes, Paris, PUF.

Laforgue, René, 1969, Psychopathologie de l'échec, Paris, Petite Bibliothèque Payot. 